\title{
Two Modes of Disc Accretion in X-ray Pulsars?
}

\author{
Ulf Torkelsson \\ Department of Astronomy $\&$ Astrophysics, Chalmers University of \\ Technology/Göteborg University, S-412 96 Gothenburg, Sweden
}

\begin{abstract}
In this paper I compare the properties of the Be/X-ray transients to those of disc accreting X-ray pulsars that show torque reversals without significant changes in the X-ray fluxes. I find that it is unlikely that the cause of the torque reversal is a radiation-induced warp in the accretion disc. The preferred explanations of the phenomenon is that either there is an intrinsic difference in the properties of the neutron stars between the two groups of objects, or that the torque reversals appear in systems in which the accretion disc is formed from a stellar wind.
\end{abstract}

\section{Introduction}

In the interaction between a magnetic neutron star and an accretion disc angular momentum is exchanged through a magnetic torque (Ghosh \& Lamb 1979). This is manifested in the variations of the spin frequencies of the X-ray pulsars (e.g. Bildsten et al. 1997). The Ghosh \& Lamb model predicts a strong correlation between the accretion luminosity and the torque acting on the neutron star, such that the larger the accretion rate the higher the spin up torque, while at very low accretion rates the torque will spin down the neutron star. This correlation is at least qualitatively in agreement with the observations of X-ray fluxes and spin variations of the $\mathrm{X}$-ray pulsars in $\mathrm{Be} / \mathrm{X}$-ray transients during their type II outbursts. However there is a group of $\mathrm{X}$-ray pulsars, which show erratic torque variations with no clear correlation between the torque and the X-ray flux. The time scale over which the torque remains constant varies between 10 to 100 days for Cen X-3 and OAO 1657-415 to several years for 4U 1626-67 and GX 1+4.

Several models have been presented to explain these torque reversals; the formation of counter-rotating discs in a stellar wind from the companion star (Nelson et al. 1997; see also Matsuda, Inoue \& Sawada 1987), a warp in the inner part of the accretion disc (van Kerkwijk et al. 1998; Lai 1999), a transition to an advection-dominated flow (Yi, Wheeler \& Vishniac 1997), or a re-structuring of the magnetic field that couples the accretion disc to the neutron star (Torkelsson 1998, Li \& Wickramasinghe 1998). In this paper I will test the models by requiring them not only to explain the torque reversals and the lack of correlation with the X-ray flux, but also to explain why the expected correlation between the torque and the X-ray flux appears in the Be/X-ray transients. In other words the intention of this paper is to look for fundamental differences in the properties of the accretion flows in these two kinds of systems. I will therefore discuss the geometry of the accretion flow in Sect. 2, and then discuss differences 


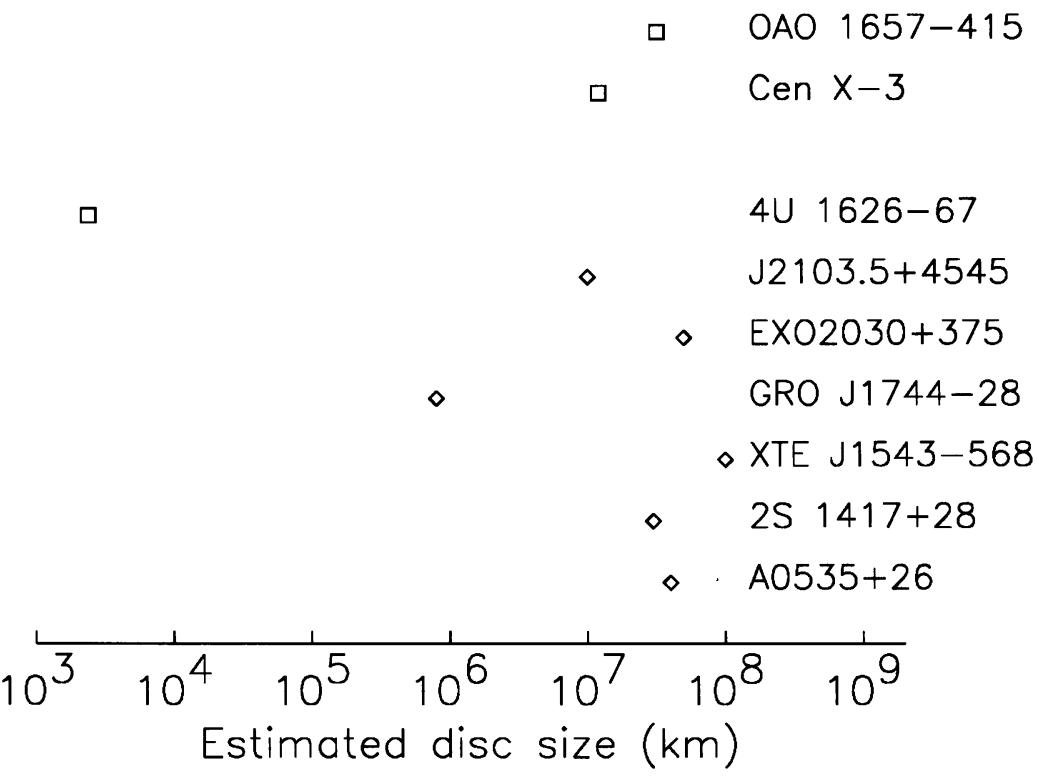

Figure 1. Estimated sizes of the accretion discs in Be/X-ray binaries (diamonds) and the X-ray pulsars showing torque reversals (squares)

in the accretion rate in Sect. 3. Finally I provide a brief discussion of my results in Sect. 4.

\section{The geometry of the accretion flow}

The formation of accretion discs has mainly been studied in the context of Rochelobe overflow in close binaries that are on circular orbits. In such systems the relative size of the accretion disc depends on the mass ratio $q$ of the two stars. For the purpose of this paper I will take the disc radius to be equal to the circularization radius. The formation of accretion discs in elliptic binaries, such as the Be/X-ray transients, is not that well understood, but as a rough estimate I will assume it to be equal to $(1-e) a_{\mathrm{X}} \sin i$, the projected minimum distance between the neutron star and the centre of mass.

There is no systematic difference in the disc sizes between the two groups of systems (Fig. 1), except that the disc is extremely small in $4 \mathrm{U} 1626-67$, which is a close binary. The disc in $4 \mathrm{U} 1626-67$ is too small for the radiation pressure to generate a warp in the disc as suggested by van Kerkwijk et al. (1998). This still leaves the possibility that an inclined magnetic field of the neutron star can produce a warp (Lai 1999).

In a pure Roche lobe overflow the velocity of the gas is small at the moment that it is leaving the companion star, which facilitates the formation of a corotating disc. As the velocity of the overflowing gas increases, for instance if the disc is formed from a stellar wind, either a co-rotating or a counter-rotating disc 


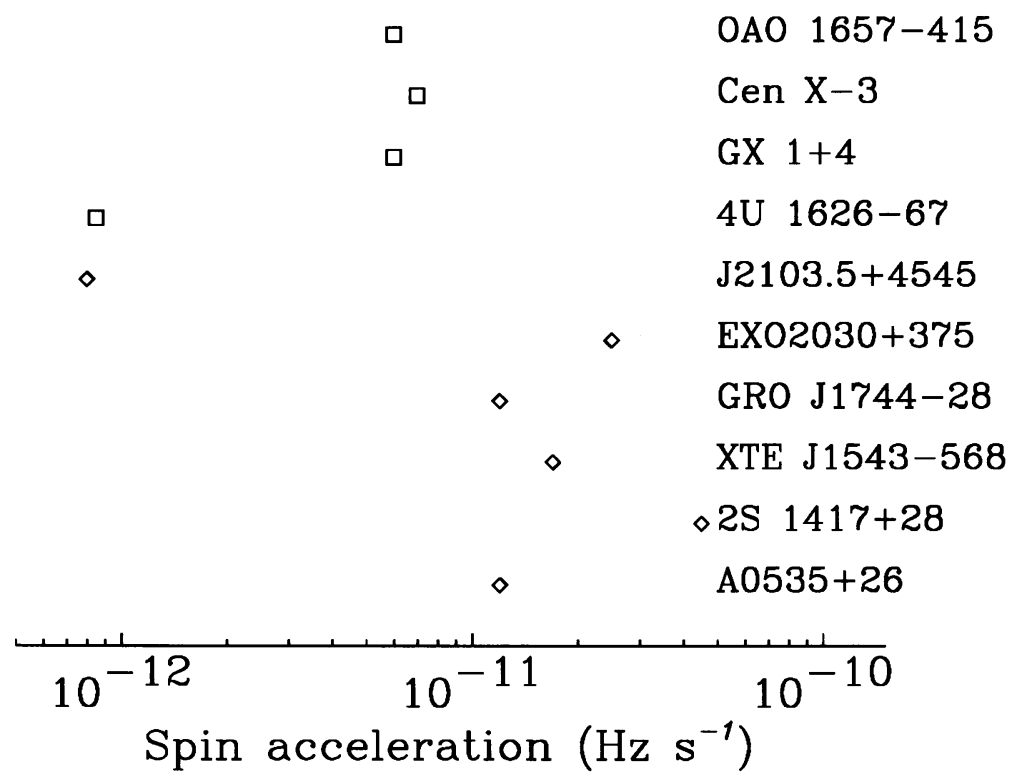

Figure 2. The maximal spin up rate for Be/X-ray transients (diamonds) and X-ray pulsars showing torque reversals (squares)

may form (Wang 1981). The outflow velocities from Be-stars are in general small $\sim 10 \mathrm{~km} \mathrm{~s}^{-1}$ (Okazaki \& Negueruela 2001). The companion stars of Cen X-3, OAO 1657-415 and GX 1+4 are all supergiants or giants that can be expected to have massive winds on the other hand. However the companion of $4 \mathrm{U} 1626-67$ is a dwarf star that cannot support a wind on its own, though there may possibly be a wind driven by the irradiation from the neutron star.

\section{Torques and accretion rates}

In the Yi et al. (1997) model the accretion disc and neutron star is close to an equilibrium in the sense that the spin up torque from the inner part of the accretion disc is almost compensated by the spin down torque from the outer part of the accretion disc. A small decrease in the accretion rate leads to that the disc changes from a thin Keplerian disc to an advection-dominated accretion flow (ADAF) which is rotating in a sub-Keplerian fashion. Since ADAFs occur at low accretion rates one can expect from this model that the maximal spin up torques should be smaller in those systems that show torque reversals than in the $\mathrm{Be} / \mathrm{X}$-ray transients. Figure 2 shows that while in general the $\mathrm{Be} / \mathrm{X}$ ray transients have higher spin up rates, the system with the smallest spin up rate is the Be/X-ray transient J2103.5+4545 (Baykal, Stark \& Swank 2002). Actually the most outstanding feature of the diagram is the low spin up rates of J2103.5+4545 and 4U 1626-67. 


\section{Discussion}

Judging from the data compiled above the cause of the torque reversals cannot be a radiation-driven warp, though the magnetic field of the neutron star may still induce a warp. This then suggests that there is a systematic difference between the orientations of the magnetic fields in the X-ray pulsar in the $\mathrm{Be} / \mathrm{X}$ ray transients and those showing torque reversals.

Another possibility is that the torque reversals appear in systems in which the accretion disc is formed from a stellar wind. The problem with this model though is to demonstrate that irradiation can generate a wind in the dwarf star of $4 \mathrm{U} 1626-67$.

The Yi et al. (1997) model in which the torque reversals are due to a transition between a thin Keplerian disc and an ADAF disc appears to be excluded since the two classes of systems show similar maximal spin up rates. It is more difficult to rule out those models that describe the torque reversals in terms of a re-structuring of the magnetic field, though Torkelsson (1998) noted that his model had problems explaining the long coherence time of the torque of $4 \mathrm{U}$ 1626-67.

Acknowledgements: This research was supported by the Swedish Research Council.

\section{References}

Baykal, A., Stark, M., \& Swank, J. H. 2002, ApJ, 569, 903

Bildsten, L., et al. 1997, ApJS, 113, 367

Ghosh, P., \& Lamb, F. K. 1979, ApJ, 234, 296

Lai, D. 1999, ApJ, 524, 1030

Li, J., \& Wickramasinghe, D. T., 1998, MNRAS, 300, 1015

Matsuda, T., Inoue, M., \& Sawada, K. 1987, MNRAS, 226, 785

Nelson, R. W., et al. 1997, ApJ, 488, L117

Okazaki, A. T., \& Negueruela, I. 2001, A\&A, 377, 161

Torkelsson, U., 1998, MNRAS, 298, L55

Wang, Y.-M. 1981, A\&A, 102, 36

Yi, I., Wheeler, J. C., \& Vishniac, E. T. 1997, ApJ, 481, L51 